\title{
Analytical Study of Deep Learning-Based Preventive Measures of COVID-19 for Decision Making and Aggregation via the RISTECB Model
}

\author{
Ishfaq Ahmad $\mathbb{D}^{1},{ }^{1}$ Sheng Jun Xu $\mathbb{D}^{1},{ }^{1}$ Amna Khatoon $\left(\mathbb{D},{ }^{2}\right.$ Usman Tariq $\mathbb{D},{ }^{3}$ Inayat Khan $\mathbb{D},{ }^{4}$ \\ Sanam Shahla Rizvi $\left(\mathbb{D},{ }^{5}\right.$ and Asad Ullah $\mathbb{D}^{2}$ \\ ${ }^{1}$ School of Information and Control Engineering, Xi'an University of Architecture and Technology, Xi'an 710055, China \\ ${ }^{2}$ Department of Information Engineering, Chang'an University, Xi'an 710064, China \\ ${ }^{3}$ College of Computer Engineering and Science, Prince Sattam bin Abdulaziz University, Al-Kharj, Saudi Arabia \\ ${ }^{4}$ Department of Computer Science, University of Buner, Buner 19290, Pakistan \\ ${ }^{5}$ Raptor Interactive (Pty) Ltd., Eco Boulevard, Witch Hazel Ave, Centurion 0157, South Africa
}

Correspondence should be addressed to Sheng Jun Xu; sjxu@xauat.edu.cn

Received 30 September 2021; Revised 4 November 2021; Accepted 15 December 2021; Published 28 January 2022

Academic Editor: Rahman Ali

Copyright (C 2022 Ishfaq Ahmad et al. This is an open access article distributed under the Creative Commons Attribution License, which permits unrestricted use, distribution, and reproduction in any medium, provided the original work is properly cited.

Coronavirus disease (COVID-19) primarily spreads through imbalanced social distancing practices. Automatic analysis is possible through deep learning-based methods to understand and control COVID-19. Healthcare analysis and prediction are best made in the situation of a pandemic such as COVID-19. This analysis can be used to classify the COVID-19 and non-COVID-19 groups and social distancing measures with good estimation by preventing immense dissemination. Postpreventive measures require parallel reinforcement to analyse current, upcoming, and uncertain situations of COVID-19 prevalence, which are effectively handled by implementing multicriteria decision-making methods. Herein, we estimate and measure the social distance by deep learning technique usage (You Only Look Once, Version 3 is a real-time object detection algorithm) in the proposed model for the analytic network process. The multicriteria decision making increases the evaluation of the risk factors. The modification of the pandemic model increases the application of social distancing and preventive measures. This model will alert us when the number of people exceeds in some area from the experimented barrier. RISTECB simulation is used in the preventive measures of the social distance among the sample population to see the initiators, infectors, suspicious, expirer, survivor, and transmitters. Postpreventive criteria used those results to set the barriers that are the critical points for prevention in uncertain situations. Therefore, this paper aimed to develop a framework, including social distancing and distance estimation, by using deep learning-based techniques through multicriteria decision-making methods such as the analytical network process. For simulation for statistical information of inclusive information of preventive measures and postpreventive measures, an automatic resonant transfer learning-based practice is used. General proportional analyses illustrate that the projected model helps in postpandemic COVID-19 preventive measures by amalgamating multiple techniques.

\section{Introduction}

Coronavirus disease 2019 (COVID-19) has become a global pandemic, and due to the prior unavailability of vaccines, detection of COVID-19 at the early stage is necessary to avoid its further spreading. The natural source of this virus is unidentified [1]. In Wuhan City, Hubei Province, China, the COVID-19 firstly broke out on December 31, 2019.
Subsequently, it spread like a fire in China and infected a group of children and juveniles and frequently originated in the old across the entire world [2]. COVID-19 was publicised as a pandemic on February 11, 2020, by the World Health Organization (WHO).

It was identified that the respiratory disease has common symptoms: cough, fever, body aching, and breathing strain [3]. Vanishing of taste, throat problems, tiredness, aches, 
and nasal impasse are found in some patients with COVID19 [4]. The interval between the incubation period and the initial indication of symptoms seemed to be 14 days [5]. Direct or indirect contact with a disease-ridden person causes the infection by the scattering of the virus through the droplets on coughing and sneezing [6]. On the other hand, vaccines or drugs for this disease are unavailable, so isolation and social distancing are the only solutions to this infection. Therefore, it is necessary to have the premature finding of disease-ridden persons to stop the scattering. Recently, vaccination has been developed and injected into the majority of the world's population. Still, total cure from this pandemic is not possible, and the danger is the variants of COVID-19. The only solution is social distance and precautionary measures even after vaccination.

$\mathrm{AI}$ and deep learning techniques enable us to diagnose various pneumonia and the COVID-19 virus as a useful screening tool. Multiple CNN models were used to classify the CXR dataset with disease and probability group of positive or negative COVID-19 [7]. Simulation was practised with new, complex, or uncommon procedures without incurring risks to the patient [8]. Individuals affected who have improved "survival in our case" later show minor or restrained indications. They are expected to develop temporary clashes to the infection and implausible to severe respirational disorders [9]. However, there have been clinical findings on rare occasions showing that patients who have recovered from the disease tested positive again. Generally, the Susceptible Exposed Infected or Recovered model was frequently used in the pandemics before COVID-19. This model was used in conditions such as SARS back in 2002 [10], the pH1N1 pandemic in 2009 [11], the MERS in 2013 [12], and the latest Ebola outbreak in 2018 [13].

A simulation begins when some pandemic influences society and creates some disturbance. In the current COVID-19 pandemic, the infected people pass the virus to nearby ones. The rate has been specified for newly born and people died due to the virus. Individuals have an age measured in a fractional number of time steps. $\mathrm{R}$ is the restrictor, $\mathrm{E}$ is the expirer, $\mathrm{S}$ is the survivor, $\mathrm{T}$ is the transmitter, $\mathrm{R}$ is recovered/removed, and $\mathrm{I}$ is the initiator. The initiatives to fight the pandemic related to social distancing are as follows: It is possible by minimising crowding in shopping malls, parks, colleges, universities, airports, and workplaces, avoiding crowds, and keeping a sufficient distance between people. Distancing oneself from others is essential, particularly for those at a higher risk of serious illness due to COVID-19. It has the potential to play a critical role in halting the spread of the virus and avoiding the pandemic's peak. Deep learning has shown promise in several everyday issues. Thanks to recent deep learning advances, object detection tasks often use these methods to calculate social distancing among people. This analytical study aims to put emphasis not only on the importance of social distancing but also on its estimation, as shown in Figure 1.

Previously, the most emphasis was on social distancing and the influential factors preventing and spreading COVID-19. MCDM methods enable selecting the already devised, modified, and new factors based on their requirement to enhance the model's capability. The elements majorly taken from the previous pandemic control preventive measures were the restrictor, expirer, survivor, transmitter, recovered, and continuer. In our work, two more factors have been added to enhance the control of pandemic spread and increase check and balance. Barrier and initiator are the two added in the existing models for preventing pandemic spread. COVID-19 is expanding and prolonging more than our expectations. Over time, it has transformed and come out with new disasters, like no other virus. However, the death rate is comparatively lower than that due to other viruses. However, its prolonged duration badly affects the economy, education, and daily routines worldwide. Barrier and initiator have been added in the model to increase the controlling capability of virus spread in pandemics, as we see in COVID-19. Distance estimation to set the barriers among suspected, initiator, and transmitter is another prominent aspect. It contributes both as a detective of infectious and maintains proper hindrances to prevent the virus's spreading. The proposed system can be implemented in the area of surveillance systems for monitoring social distancing. Due to COVID-19, threats are in daily life where crowds create a social distancing imbalance.

Deep learning in social distancing implementation can be helpful in terms of monitoring social distancing imbalance, besides the institution sector, government sector, shopping malls, and crowded areas of the daily circle of human gathering. The simulation part is an efficient monitoring system that conceals and investigates future circumstances. Statistically, monitoring can be efficient through the RISTECB model in common day-to-day life routines. The healthcare centre, individual territory, and the government can easily tune the parameter according to the natural environment spreading of COVID-19. It helps monitor the common daily life routines to control pandemic spreading and added circumstances through tuning the parameters. Every healthcare centre, territory, and government sector examines the preventive and postpreventive measures through an efficient RISTECB model. The easily tunable parameters are helpful in every system in the government to small monitoring centres of the common day-to-day life routines.

\section{Materials and Methods}

2.1. Multicriteria Decision Making for Social Distancing. Social distancing has a practical approach to diminishing physical interaction among potentially diseased persons and healthy inhabitants. According to the rules of the WHO [14], 6 feet of distance is necessary to follow social distancing. In many countries, such as KSA (Kingdom of Saudi Arabia), 2 meters distancing is followed. Social distancing is an important containment measure because corona infection might be unintentionally transmitted to others [15]. Appropriate social distancing diminishes the infection percentage of viral diseases from $60 \%$ to $90 \%[16,17]$. Infection diseases' increase from the initial to peak level can be considered a condensed peak; this condensed peak may 


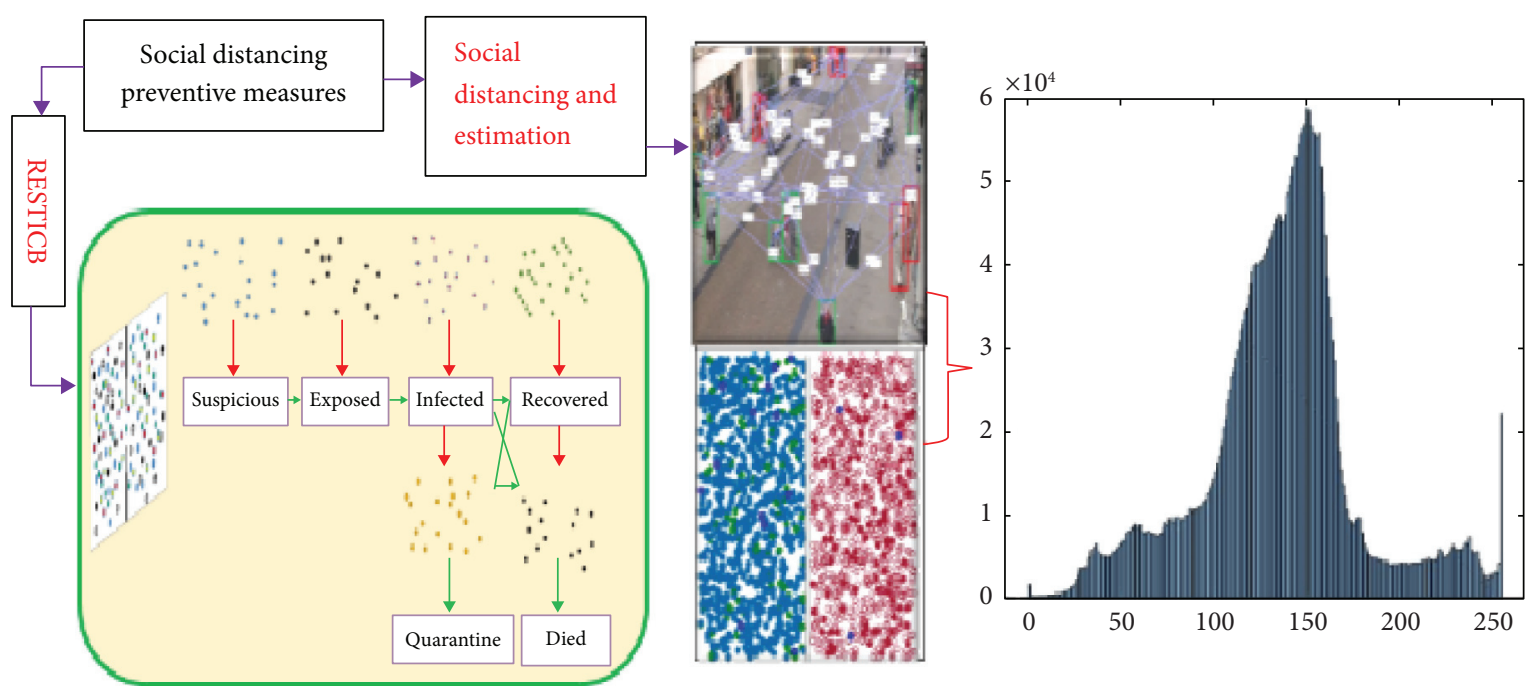

Figure 1: Social distancing with deep learning accumulated along with the simulation.

confidently tie to the accessible healthcare structure. The benefits, as mentioned earlier, deal with healthier amenities to the diseased persons fighting in the inconsistency of the pandemic [18]. The study of epidemiology concerns influences and aims for spreading of infectious viruses.

Epidemiology is a detailed study of the spread of infectious diseases by knowing the factors and reasons. Social distancing is an appropriately consistent method to control and prevent the pandemic [19]. Undoubtedly, those social distancing measures had been enacted in China to regulate COVID-19 at first, then accepted, and adopted worldwide later. Prem et al. [20] studied the effects of social distancing measures by consuming synthetic location-specific contact patterns. It is about the continuing route of the epidemic, expending "susceptible" cases who "exposed" initial symptoms or "infected" avoid social distancing and "removed" can be dead person (SEIR) models. Untimely and unexpected chaotic indistinct barriers are placed where the rate of infectious disease is relatively high. There is a chance of high prevalence or lockdown of social distancing, leading to a former inferior peak. An inferior peak is when preventive measures are slowed down due to the minimal cases around. This situation can be flattened by reducing interventions, e.g., perception of minimal risks in case of fewer cases, governmental check balance leniency, and gradual awareness of hidden future problems [21].

It is a fact that social distancing is essential for pandemics but economically painful. It has to be performed to flatten the infection curve as observed in COVID-19 control. Adolph et al. [22] stated that due to lack of understanding among all in the United States of America, people prefer their economic advantages and negate the preventive measures in the form of social distancing causing great destruction. Implemented policies adopted initially resulted in the ongoing influence in the society towards the pandemic. Consequently, social distancing influences commercial production directly and indirectly. See Figure 2 to observe and study to prevent death rate and infections. Walters et al. [23] researched social distancing and economic

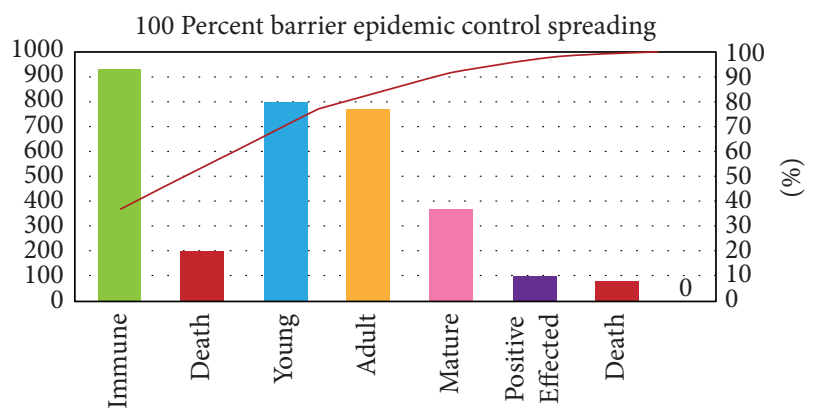

FIGURE 2: The barrier is set as 100 percent to preventing death rate and infections where open border increases the mortality rate, while putting a barrier on $100 \%$ reduces the tremendous preventing results for mortality and spreading rate control.

status. The graph showed that transitional levels of activities were allowed while avoiding a massive outburst. These results showed the flat death rate age-wise and infection reasons, e.g., immune and positive affected.

Respiratory diseases are contagious as the rate and mode of spreading viruses are the most critical factors. Social distancing, economic stability, and essentials provided by the government on a priority basis are essential steps to spread the virus publicly. Besides most laboratories, scientists, doctors, researchers, and multidisciplinary medication organisations pay attention to proposing or making vaccines to prevent COVID-19. Still, there are no vaccines and medicines for its treatment. Hence, precautionary steps [24] suggested a transformed SIR model and a social distancing measure performance parameter. Suspicious cases are denoted as $\mathrm{S}$, and I indicates the infectious persons, as they both are the key elements of analysis to prevent pandemics such as SARS and COVID-19. R showed that recovered persons successfully study $S$ and I cases by adopting the necessary preventive measures.

Multicriteria decision making is effective since pandemic dynamics are highly nonlinear, and preventive interventions can have unintended consequences. In traditional epidemic 
models, human behaviour is unaffected by the outbreak and stable over time [25]. The Kermack-McKendrick compartmental epidemic model assumes that compartment sizes are big enough to provide homogenous mixing of individuals or at least homogenous mixing in each subgroup if activity levels stratify the population. While it is widely acknowledged that humans do take precautions during an attack, models that integrate behavioural dynamics are typically much more challenging to analyse. Since infection is spread by the interaction between susceptible and infectious individuals, the population's contact pattern is a vital modelling component. Most models that consider behavioural responses to disease dynamics are relativistic due to their study difficulties. These issues revolve around an individual's elevated anxiety levels concerning their health, the health of their peers, certain avoidance behaviours as a result of the sickness, and behavioural changes in the affected community. Analytical network process is implemented here to evaluate the selection of models in behavioural dynamics.

COVID-19, a novel coronavirus, is a respiratory pandemic. Despite many studies on this fast-spreading pandemic, few use MCDM approaches. Majumder et al. [26] suggested a new TOPSIS technique for COVID-19 risk factor identification. Instead of Euclidean distance, the recommended method uses the first-time supremum metric. Mohammed et al. [27] created an integrated MCDM technique to evaluate the multiple COVID-19 diagnostic models. The proposed method uses entropy to determine criteria weights and TOPSIS to rank COVID19 diagnostic models. SpNoF sets are a new idea developed by Yang et al. [28]. The study develops a new score, accuracy function, and the SpNoF and weighted Bonferroni mean operators. Based on the suggested SpNoF operators, a multicriteria decision-making technique for antivirus mask selection is devised. Sensitivity and comparison analyses are used to assess the proposed method's suitability. During the COVID-19 epidemic, a program was created in Singapore to determine if two people were related [29]. Bluetooth technology in their smartphones allows them to communicate with each other.

In the same way, in South Africa, security camera video, facial recognition technology, and a global location system have all been used in Korea [29]. The Global Positioning System (GPS) was used to offer real-time data for contact tracing. The most efficient utilisation of big data technologies in Taiwan is a significant reason for the country's low number of infected and affected people deaths [30]. When formulating guidelines, these relationships might be considered for further investigation, prevention, and control of pandemics.

This paper studies a network RISTECB (recovered-infectious-susceptible-transmitter-effected-continuer-barrier) pandemic with preventive social distancing. We consider the setting where susceptible, affected, initiator, and continuer all as individuals distance themselves from their neighbours and other places through barriers and social distance estimation. We explore the effects of social distancing on the
YOLOv3 and RISTECB models in real-world networks. Depending on the populations' network structure, social distancing could boost the pandemic threshold parameter from below to above its threshold value, allowing for a large outbreak where one would not otherwise be feasible. Our study of theoretical results stresses the importance of using preventive steps in pandemic models.

\section{Methods}

This part illustrates the proposed a multitask framework for social distancing imbalance, expending the deep learning approach of YOLOv3; simulation-based statistical analysis for preventive and postpreventive measures, as shown in Figure 3. A comparative study of a different detection model and analysis to maintain social distancing in some densityestimated zones is based on ANP.

(i) Social distancing simulation preventing immense dissemination

The lockdown feature that separates half of the infected and noninfected people covers social distancing. The lockdown features separate the clusters of infected and noninfected people; the RISTECB model is enhanced to protect the population length and can be a separated barrier on the high menace area.

(ii) Statistical model helpful with the pretuned model to predict the better results than the SIR model.Results are based on the model's tuning, surveillance rate, mortality, spreading, population, restricted area, and distance per infected rate.

A deep learning model helps distinguish the people walking along or meeting together. Deep learning measures social distancing, distance estimation, and social distancing. The imbalance highlight risky distance is real-time data implementation on the old Oxford mall dataset and our new dataset (https:// exposing.ai/oxford_town_centre/; UET person data).

\section{YOLOv3}

SSD and YOLOv3 (YV3) are mainly used for object detection, and Yv3 is considered more competitive than SSD in terms of real-time detection. The sample criteria collected based on manner can expect the sort and locality of an object that only observes once at the image. Yv3 in Figure 4 deliberates the regression task as a substitute for classification to allocate labelled likelihoods to the rectangle of a person. A particular convolutional neural network simultaneously calculates numerous boxes and class probabilities. Yv3 does multilabelled classification supported by logistic classifiers. Yv3 produces three foresights for the individually spatial locality at diverse scales in an image that excludes the problem of not perceiving minor objects proficiently [31]. Each estimate is supervised by calculating the objects, Box regressor, and classification scores. Figure 4 illustrates the description of the "Yv3" architecture. 


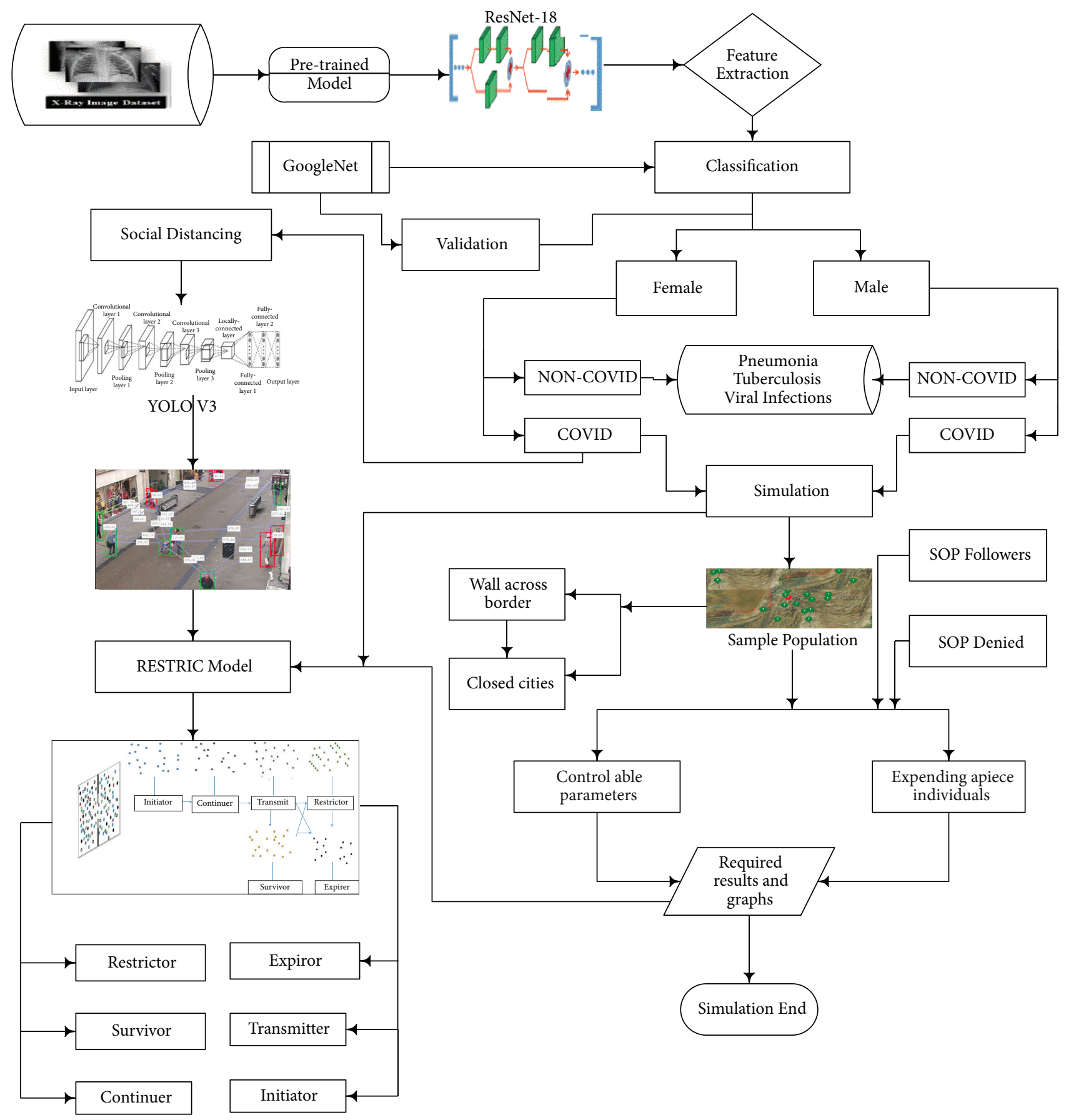

Figure 3: Framework for COVID-19 detection, simulation, and discrimination.

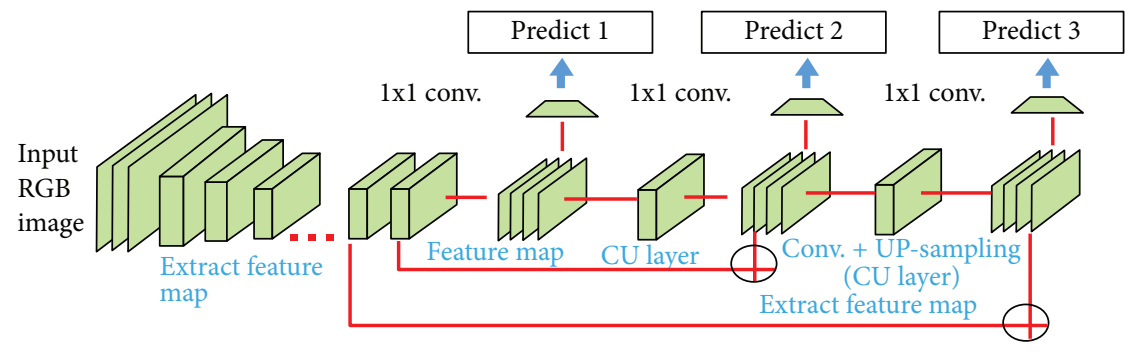

Figure 4: YOLOv3 object detection architecture. 
4.1. Deep Sort. In the contemporary study, a deep sort was exploited to track characters contemporaneous in the surveillance tape. Patterns learned marked usage via detected objects in the images. That has advanced collective through the sequential information for expecting allied arcs of the things of interest. It retains a trail of individual object attention by plotting unique identifiers additional statistical and probabilistic analysis. The Hungarian algorithm (HAG) adds connotation and id description that classify existing objects in the prevailing frame compared with the previous one. Primarily, FRCNN is proficient in identifying an individual, tracing a linear continuous velocity model [32]. The deep sort is also worthwhile holder-related encounters such as obstruction, numerous perspectives, and nonimmovable cameras. The Kalman filter (KF) and the HAG have been utilised for efficient tracking. Though KF is recursively rummage-scale for the enhanced association, it can envision imminent logic grounded in the exact position.

4.2. ANP (Analytical Network Process). During a pandemic, most people's attention is drawn to whether or not the disease has spread. Health precautions assigned to them might produce a safe and healthy environment. Initially, we should consider meeting sanitation and health needs and make design recommendations. The fundamental cause for this, in our view, is when people's basic needs, such as health and safety, are jeopardised. As a result, in a pandemic, these necessities should take precedence. More importantly, clever technology-integrated methods have been successful. Compared to the Analytic Hierarchy Process (AHP), the Analytic Network Process (ANP) is a multicriteria theory of measurement used to prioritise absolute results from individual assessments [33].

The following are the basic steps for using ANP:

(1) A set of hierarchical network models is created that addresses a given problem is put together

(2) Datasets are used to make pairwise comparisons

(3) The internal dependencies establish all pieces' general priority by constructing a supermatrix at each stage level

(4) Judgments are made based on the information you have gathered

Pandemics have posed severe hazards to global health and public safety in modern society. Societal order and security are important although humans are capable of defeating pandemics. Despite widespread vaccination and the use of antiviral medications, we cannot prevent the spread of the virus. In the event of the next pandemic, as a result, research into pandemic prevention and control is essential. It is a popular topic all the time.

4.3. Mahalanobis Distance Formula. The Hungarian approach was used in the directive to handle the challenge of mapping between new inward dimensions. It begins by arranging data into a matrix, with persons acting as rows and actions as columns. Kalman filtering is a technique for estimating unknown variables based on observations collected over time. Making a distinction in equation (1) between appearance and motion determined by Mahalanobis yields the expected Kalman states [34].

$$
d^{(1)}(i, j)=\left(d_{j}-y_{i}\right)^{T} S_{i}^{-1}\left(d_{j}-y_{i}\right)
$$

Prognostication of the $i^{\text {th }}$ track scattering preoccupied with dimension space is signified by $\left(y_{i}, S_{i}\right)$ DJ and the bounding box (BB) detection. The Mahalanobis distance deliberates this improbability by approximating the sum of standard deviations and the mean track position away for detection [34]. The framework has to measure the distance, social distancing, imbalance, and estimation of FRCNN from the infected and disinfected territory. Additionally, by thresholding the Mahalanobis distance, this metric eliminates the unlikely associations [34]. This verdict signified with a target that appraises equation (1), finding the existence association among the $\left(i^{\text {th }}, j^{\text {th }}\right)$ detection is admissible in.

$$
b_{i, j}^{(1)}=1\left[d^{(1)}(i, j)<t^{(1)}\right] \text {. }
$$

However, possible camera indication affects Mahalanobis distance enactment additional metric [35] that estimates the minor cosine distance amid the $i^{\text {th }}$ track and $j^{\text {th }}$ detection as in equation (3) explained:

$$
d^{(2)}(i, j)=\min \left\{1-r_{j}^{T} r_{k}{ }^{(i)} / r_{k}{ }^{(i)} \epsilon^{2}\right\} .
$$

Again, a binary mutable is presented to designate if an association is tolerable [36]:

$$
b_{i, j}^{(1)}=1\left[d^{(2)}(i, j)<t^{(2)}\right] .
$$

An appropriate threshold is an extent for this pointer on a distinct training dataset. The association problem with equal metrics in dimension combined with equation (5) using a weighted sum is [37]

$$
b_{i, j}^{(1)}=1\left[d^{(2)}(i, j)<t^{(2)}\right] \text {. }
$$

Association is permissible ambiguity within the gating section of equal metrics, as follows:

$$
b_{i, j}=\prod_{m=1} 2 b_{i, j}^{(m)} .
$$

The encouragement of the respective metric on the mutual association rate can control the hyperactive parameter $\lambda$. YOLO $\mathrm{v} 3$ consists of localisation loss with bounding box regressor, cross entropy, and confidence loss for classification score, defined in equation (7). The localisation loss measures the predicted boundary box locations and sizes errors. We only count the box responsible for detecting the persons. 


$$
\lambda_{\text {coord }} \sum_{i=0}^{S^{2}} \sum_{j=0}^{B} \text { person }\left[\left(t_{x}-\widehat{t}_{x}\right)^{2}+\left(t_{y}-\widehat{t}_{y}\right)^{2}\right]+\lambda_{\text {coord }} \sum_{i=0}^{S^{2}} \sum_{j=0}^{B} i j \text { person }\left[\left(\sqrt{t_{w}}-\sqrt{\widehat{t}_{w}}\right)^{2}+\left(\sqrt{t_{h}}-\sqrt{\widehat{t}_{h}}\right)^{2}\right],
$$

where $\sum_{i=0}^{S^{2}} \sum_{j=0}^{B}{ }_{i j}^{\text {person }}$ if the $j$ th boundary box in cell $i$ is responsible for detecting the person, otherwise $0 . \lambda_{\text {coord }}$ is the increase in the weight for the loss in the boundary box coordinates.

If an object has been detected in the box, the confidence loss (measuring the objectness of the box) is

$$
\sum_{i=0}^{S^{2}} \sum_{j=0}^{B} \operatorname{person}_{i j}\left(C_{i}-\widehat{C}_{i}\right)^{2}
$$

where $\widehat{C}_{i}$ is the box confidence score of the box $j$ in cell $i$. $i_{i j}^{\text {person }}=1$ if the $j^{\text {th }}$ boundary box in cell $i$ is responsible for detecting the person, otherwise 0 . If an object did not detect in the box, the confidence loss is

$$
\lambda_{\text {no_person }} \sum_{i=0}^{S^{2}} \sum_{j=0}^{B}{ }_{i j} \text { no_person }\left(C_{i}-\widehat{C}_{i}\right)^{2} \text {, }
$$

where ${ }_{i j}^{\mathrm{no}_{\mathrm{p}} \text { erson }}=1$ is the compliment of ${ }_{i, j}^{\text {person }} . \widehat{C}_{i}$ is the box confidence score of the box $j$ in cell $i$. $\lambda_{\text {no_person }}$ is the weighdown of the loss when detecting background. If a person has been detected, the classification loss at each cell is the squared error of the class conditional probabilities for each category:

$$
\sum_{i=0}^{S^{2}} \text { person } \sum_{c \in \text { classes }}\left(p_{i}(c)-\widehat{p}_{i}(c)\right)^{2},
$$

where ${ }_{i}^{\text {person }}=1$. If a person appears in cell $i$, otherwise 0 . $\widehat{p}_{i}(c)$ denotes the conditional class probability for class $c$ in cell $i$. The final loss adds localisation, confidence, and classification losses together.

$$
\begin{aligned}
& \lambda_{\text {coord }} \sum_{i=0}^{S^{2}} \sum_{j=0}^{B} i_{j}^{\text {person }}\left[\left(t_{x}-\widehat{t}_{x}\right)^{2}+\left(t_{y}-\widehat{t}_{y}\right)^{2}\right]+\lambda_{\text {coord }} \sum_{i=0}^{S^{2}} \sum_{j=0}^{B} i_{j}^{\text {person }}\left[\left(\sqrt{t_{w}}-\sqrt{\widehat{t}_{w}}\right)^{2}+\left(\sqrt{t_{h}}-\sqrt{\widehat{t}_{h}}\right)^{2}\right] \\
& +\sum_{i=0}^{S^{2}} \sum_{j=0}^{B} i_{j}^{\text {person }}\left(C_{i}-\widehat{C}_{i}\right)^{2}+\lambda_{\text {no_person }} \sum_{i=0}^{S^{2}} \sum_{j=0}^{B}{ }_{i j}^{\text {no_person }}\left(C_{i}-\widehat{C}_{i}\right)^{2}+\sum_{i=0}^{S^{2}}{ }_{i j}^{\text {person }} \sum_{c \in c \text { lasses }}\left(p_{i}(c)-\widehat{p}_{i}(c)\right)^{2} \\
& \Rightarrow \lambda_{\text {coord }} \sum_{i=0}^{S^{2}} \sum_{j=0}^{B} i_{j}^{\text {person }}\left[\left(t_{x}-\widehat{t}_{x}\right)^{2}+\left(t_{y}-\widehat{t}_{y}\right)^{2}\right]+\left[\left(\sqrt{t_{w}}-\sqrt{\hat{t}_{w}}\right)^{2}+\left(\sqrt{t_{h}}-\sqrt{\hat{t}_{h}}\right)^{2}\right] \\
& +\sum_{i=0}^{S^{2}} \sum_{j=0}^{B} i_{i j}^{\text {person }}\left(C_{i}-\widehat{C}_{i}\right)^{2}+\lambda_{\text {no_person }} \sum_{i=0}^{S^{2}} \sum_{j=0}^{B}{ }_{i j}^{\text {no_person }}\left(C_{i}-\widehat{C}_{i}\right)^{2}+\sum_{i=0}^{S^{2}}{ }_{i j}^{\text {person }} \sum_{\text {ceclasses }}\left(p_{i}(c)-\widehat{p}_{i}(c)\right)^{2} .
\end{aligned}
$$

Equation (11) defines the localisation loss measures with errors in the predicted boundary box. It counts the box responsible for detecting the persons, and an object did not detect. The classification loss at each cell is presented in the equation. The squared error with the class conditional probabilities if a person has been detected is observed. The final loss adds localisation, confidence, and classification losses together.

\section{Simulation}

We are modelling COVID-19 using simulation by selecting the following parameters in Table 1 according to the requirement of our proposed model.

$$
\begin{aligned}
& \frac{\mathrm{d} S(t)}{\mathrm{d} t}=\Lambda-\mu S(t)-\beta(1-\sigma) S(t) I(t)+R(t), \\
& \frac{\mathrm{d} E(t)}{\mathrm{d} t}=\beta(1-\sigma) S(t) I(t)-(\mu+\alpha) E(t), \\
& \frac{\mathrm{d} I(t)}{\mathrm{d} t}=\alpha E(t)-(\mu+\gamma) I(t)-\Delta I(t), \\
& \frac{\mathrm{d} R(t)}{\mathrm{d} t}=\gamma I(t)-\mu R(t)-R(t), \\
& \frac{\mathrm{d} D(t)}{\mathrm{d} t}=\Delta I(t), \\
& \frac{\mathrm{d} B(t)}{\mathrm{d} t}=\{\mathrm{d} D, \mathrm{~d} I \& \mathrm{~d} R\} .
\end{aligned}
$$


TABle 1: Parameters of simulation.

\begin{tabular}{lcc}
\hline Parameters & Symbols & Parameters \\
\hline Constant COVID-19 genetic frequency in the halves crowed & $\Lambda$ & Initiator suspicious \\
Death frequency circumstances other additional COVID-19 & $\mu$ & Exposed transmitter \\
The frequency of an unprotected individual becomes infected & $\alpha$ & Transmitter infected \\
Frequency per S and I contact of transmission & $\beta$ & Survivor previous infected \\
Frequency of recovered & $\gamma$ & Expirer as dead \\
Progression rate of asymptomatic/symptomatic individuals to confirmed & $\tau$ & Barrier as prevention \\
Transmission rate from asymptomatic to symptomatic & $\sigma$ & The entire population in the full or halves $\quad N(t)$ \\
The transition rate of infected individuals & $\Delta$ & $D(t)$ \\
\hline
\end{tabular}

It is established that

$$
S(t)+E(t)+R(t)+D(t)=N(t)
$$

$\tau_{\text {infected }}=1 / \alpha ; \tau_{\text {recovered }}=1 / \gamma$. The time spent isolated or infected in fatal cases is $\tau_{\text {isolated }}=1 / \delta$. In this paper, we establish that $\tau_{\text {infected }}=\tau_{\text {recovered }}=\tau_{\text {isolated }}$. We suppose that patients devote a similar sum of time to inaccessible or transmitter, whether they survivor from the group of COVID-19. That can be resolute with the aid of the number of recovered and infected persons signified as $\mathrm{R}$ and $\mathrm{I}$, individually in existing, while in our work, we modified the model by including the following more attributes in that existing model. $\mathrm{I}=$ initiator, $\mathrm{C}=$ continuer, $\mathrm{R}=$ restrictor, $\mathrm{T}=$ transmitter, $\mathrm{S}=$ survivor, $\mathrm{E}=$ expirer, $\mathrm{B}=$ barrier, and the population is $\mathrm{N}=\mathrm{C}+\mathrm{S}+\mathrm{I}+\mathrm{R}+\mathrm{E}$ and infection rate $=\alpha$ and recovery rate $=\beta$.

$\left(a(I, R)^{2} \in[0,1]\right) \longrightarrow S \longrightarrow I$ is denoted as a social distancing term, and we have

$$
\begin{aligned}
\frac{\mathrm{d} S}{\mathrm{~d} t} & =-\alpha S \frac{I}{N} a(I, N), \\
\frac{\mathrm{d} I}{\mathrm{~d} t} & =-\beta I+\alpha I \frac{I}{N} a(I, N), \\
\frac{\mathrm{d} R}{\mathrm{~d} t} & =\beta I
\end{aligned}
$$

where $a=(1-(I+R / N))^{k}$ is a long-term awareness, $k>0$ is the behaviour parameter, $a=(1-(I / N))$ is a short-term awareness, and $k>0$ is a behaviour parameter.

The advanced worth of $k$ indicates that personages are suitable to the pandemic pervasiveness.

\section{Result and Analysis}

The appearance of deep learning has carried the finest performing practices for a wide diversity of tasks. These challenges include medical diagnosis, speech recognition, and machine translation. Most tasks involve object classification, recognition, detection, tracking, and segmentation. The convolution neural network (CNN) proposed architectures have near to perfect performance. That is the key and basic architecture for object detection for excellence achievement. In the present article, COVID-19 cases thrive, object detection, and tracing models to help social distancing. YOLO v3 in the model is the stability of accuracy and speed, with the deep sort for object detection. For monitoring social distancing and estimation in pedestrian detection through surveillance footage of Oxford Town Center (OTC). MATLAB 2019a is used to test robustness, while the testing set is also convoyed through the frames of surveillance footage of the (OTC). In YOLOv3, the images are scaled to fix in a dimension of $D \times D$ with a $D$ value of 416. This footage has congruently used to achieve and simulate in observing the social distancing.

6.1. Social Distancing and Distance Estimation. The mAP and the localisation were used to obtain the models' performance throughout the training segment. It is continuously monitored and classified, and an overall loss in the person's detection is as in Figure 5. The outcomes of a YOLOv3 model attained the concluded training segment with the number of iterations (NoI) per epochs, training time (TT), mAP, and total loss (TL) value. Though the faster ResNet18 model attained minimal loss with maximum mAP, it has the lowest FPS, making it inappropriate for simultaneous uses. Likewise, compared to SSD, YOLO v3 accomplished enhanced results with composed $\mathrm{mAP}$, training time, and FPS score. The YOLO v3 model was then used to monitor the social distancing on the surveillance video. While distance estimation achieved tremendous social distancing results, in Figure 5, each social distancing group has outstanding results with corresponding objects or neighbourhood objects. The distance counts each group of items where the red rectangle is also known as a threatening. A single person is crossing along as a neighbour; however, the red rectangle (threatening) group also associated with red line distance estimation is known as the congested red line (CRL) and blue line distance estimation is known as a preventing blue line $(\mathrm{PBL})$.

Computer vision classification testing on the Oxford Town centre dataset among the street view in the first phase in Figure 5 in the 100 meters per cubic people classifies the persons' social distancing where the distance reaches the maturity of distance to find out the persons per 102 per meter and distinguish the people to measure proper distancing. The real-time implementation of data testing (UET Peshawar, Pakistan) in Figure 6 with real-time data footage shows good results under the area of persons per 102 per meter.

6.2. Controlling the Parameter for Social Distancing. The model has YOLO v3 with real-time testing and implementation for training and testing $80: 20 \%$ simultaneously. 

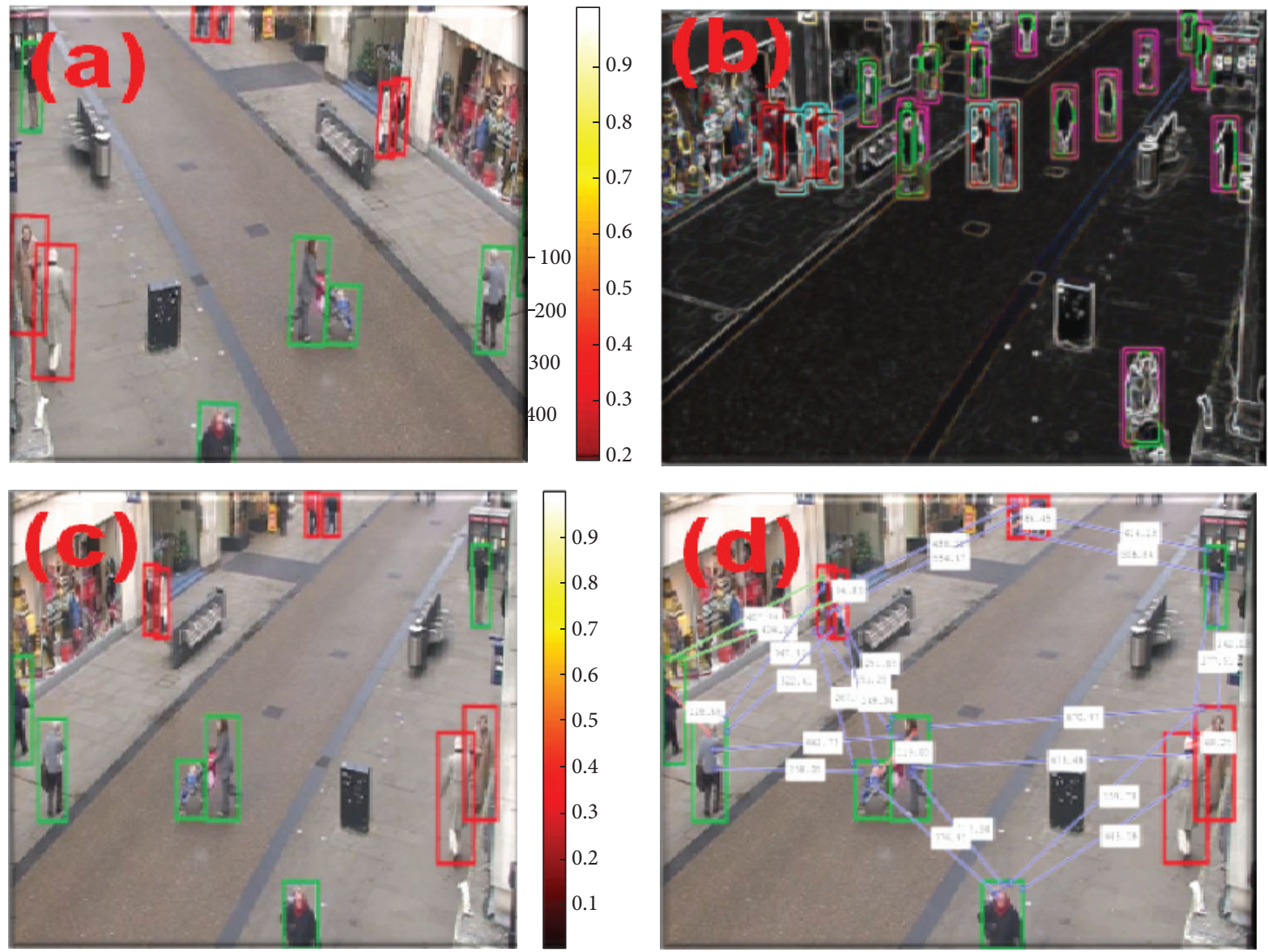

FIgURE 5: Social distance measurement and estimation using YOLOV3 and Mahalanobis distance formula: (a), (b), (c) Red and green boxes to show danger and safety, respectively. (d) Measuring the distance among groups of people distance formula with distinguishing CRL and PBL.

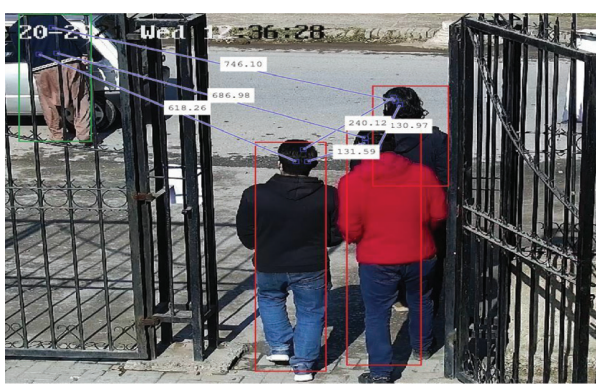

(a)

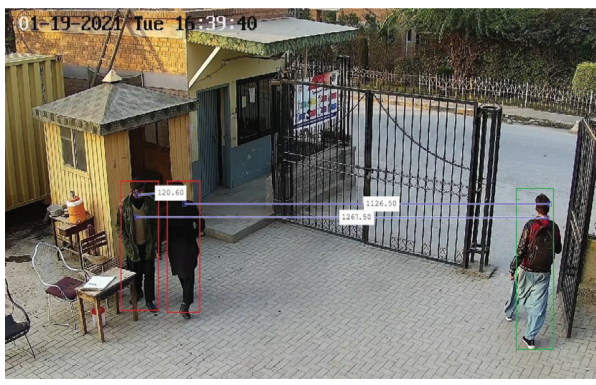

(c)

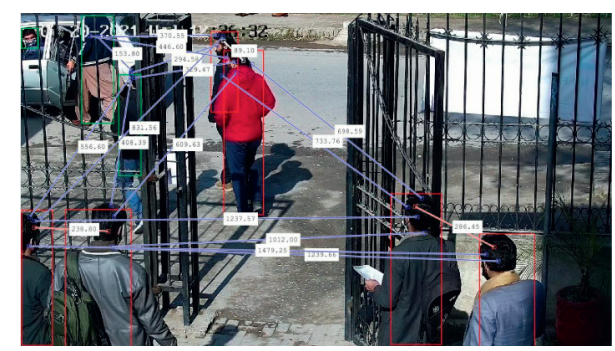

(b)

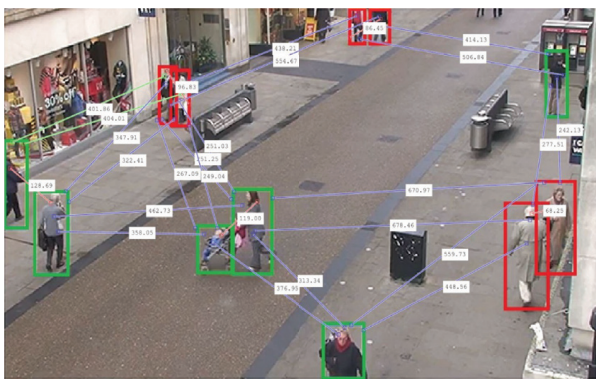

(d)

FIGURE 6: Social distance measurement and estimation using YOLOV3 and Mahalanobis distance formula: (a), (b), (c) Red and green boxes to show danger and safe on the UET dataset, respectively. (d) Measuring the distance among groups of people distance formula with distinguishing on Oxford Town Center. 
The real-time testing and performance are carried out on both datasets (Oxford Town Centre and UET Peshawar). The real-time implementation of UET and distance estimation is based on the Mahalanobis formula, which comparatively monitors the result of every individual participating in the testing phase, while every individual is making sense to maintain the social distance and social distancing violation examined. The proposed framework uses deep learning, the YOLOv3 model, and the data augmentation method to get the desired database images. YOLOv3 is a highly efficient architecture that can apply to edge devices with limited computing power. The splitting of a database for training and testing is $80 / 20$; each image is inevitably converted into $224 * 224 * 3$. The initial learning rate is $1 e-3$, the number of Epochs is 60 , and the batch size is 6 of the training database. These are the primary training and controlling parameters to tune the efficiency of network architecture. If we increase or decrease these three parameters, it will affect the results of the proposed method. In Figure 6, we can observe the adequate accuracy and elaborated distance estimation and distancing.

The moderate and wrong social distancing in our purpose-based model testing results is in Figure 7. It identifies different human pose estimations (behind the barrier (b), (c) occlusion, or a person fully covered). This section includes the necessary steps to compose a framework for monitoring social distancing. We fine-tune the trained object detection model to identify and track the person in the footage. The trained model is needed with the surveillance footage. The model generates a set of bounding boxes and an ID for each identified person. Each individual is associated with threedimensional feature space $(x, y, d)$, where $(x, y)$ corresponds to the centroid coordinates of the bounding box and $d$ defines the depth of the individual as observed from the camera.

$d=((2 * 3.14 * 180) /(w+h * 360) * 1000+3)(14)$, where $w$ is the width of the bounding box and $h$ is the height of the bounding box [38]. For the bounding boxes, the pairwise L2 norm is computed as follows:

$$
\|D\|_{2}=\sqrt{\sum_{i=1}^{n}\left(q_{i}-p_{i}\right)^{2}},
$$

where $n=3$.

The dense matrix of the L2 norm is then utilised to assign the neighbours for each individual that satisfies the closeness sensitivity. With extensive trials, the closeness threshold is updated dynamically based on the person's spatial location in a given frame ranging between $(90,170)$ pixels. Any individual who meets the closeness property has assigned a neighbour or neighbours forming a group represented in a different colour-coding compared to other people. The formation of groups indicates the violation of the practice of social distancing. That is quantified with the help of the following.

We consider ng as the number of groups or clusters identified and $n_{p}$ as the total number of people nearby. $v_{i}=n_{p} / n_{g}$, where $v_{i}$ is the violation index.
6.3. Parameter Sensitivity and Tracker Analysis. CNN-SVM is a CNN-based tracking method with robust features but lacks temporal information to deal with severe occlusion. YOLO with Kalman filter considers the temporal evolution of locations while ignorant of actual environments. Due to fast motions, occlusions, and occasional imperfect detections, YOLO with the Kalman filter performs inferiorly, lacking knowledge of the visual context. In contrast, with LSTM, YOLO synthesises over sequences the robust image features and their soft spatial supervision. YOLO is spatially resonant, as it can interpret the visual elements and detect objects on its own. It is also temporally deep by exploring temporal features and their possible locations. Step size denotes the number of previous frames considered each time for a prediction by modified YOLO the overall performance and running time; we repeat the second experiment with various step sizes and illustrate the results in Figure 8.

The proposed method was performed to achieve better accuracy than other methods mentioned in the comparative analysis with different methodologies. At the same time, no one implements real-time data and environment, and the proposed method comparative result analysis on the other state-of-the-art model is shown in Table 2. Social distancing monitoring distance estimation and people detector testing on a different real-time dataset is still needed to find results and implement a real-time dataset (UET Peshawar, Oxford Mall dataset).

The proposed method achieved $97.7 \%$ accuracy on social distancing and gained $97.88 \%$ on the distance estimation, and the real-time implementation testing achieved 95.44, which is comparatively higher than that of another state-ofthe-art model. Keniya et al. [39] focused on the social distancing Net-19 developed by them to detect a person's frame. Its displayed labels achieve an accuracy of $92.8 \%$ within a certain distance [40]. Yadav et al. [41] proposed two-person interaction MIE without limiting COVID-19 achievements or social distancing. It is based on $\mathrm{HOG}$ features to achieve 93.3\%. Escalera et al. [42] proposed a method that presented an intelligent surveillance system for people tracking and social distancing classification. It is based on thermal images with safe and unsafe COVID-19 violence. It achieved a 95\% result on Faster R-CNN. However, the real-time implementation is missing in this case. Nevertheless, each pair's real-time implementation and distance in real time are missing. Our proposed method achieved massive results, about $97.5 \%$ using our own annotated database.

UET Peshawar and publicly available databases (Oxford Town Centre) use YOLO v3 architecture. Additionally, the distance estimation for each pair of social distancing estimation can be monitored. The proposed method is fair than other comparative methods, and it achieves promising results of $97.5 \%$ with different aspects as in Table 2. In our literature review, it was seen that no researcher focuses explicitly on the social distancing with each pair of distance estimation that helps for the fair social distancing monitoring. The proposed method used different datasets for training and testing our social distancing handmade dataset 


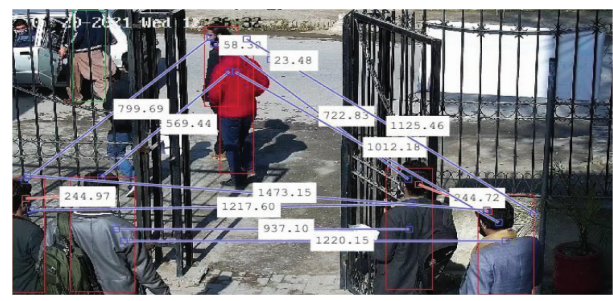

(a)

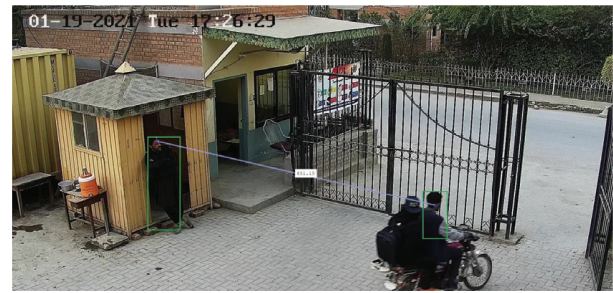

(c)

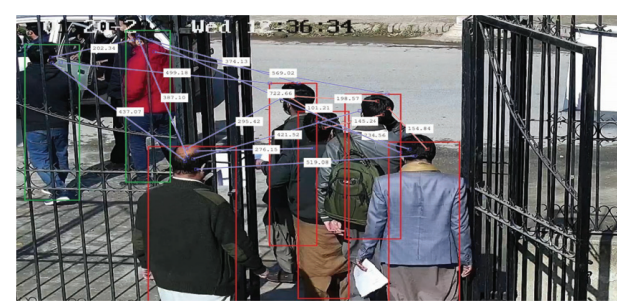

(b)

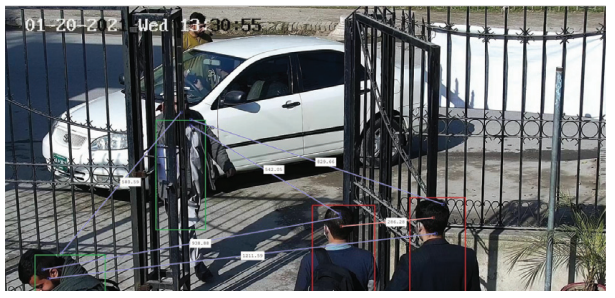

(d)

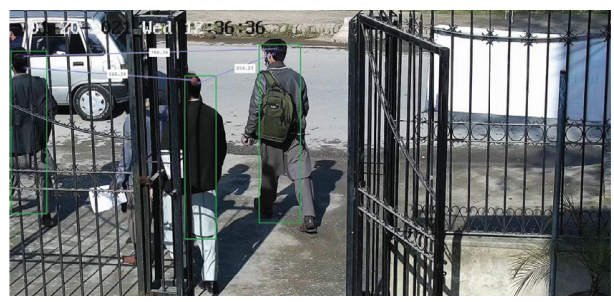

(e)

FIGURE 7: Low and moderate social distance measurement and estimation using YOLOV3: (a), (b) Moderate UET datasets. (c), (d), (e) Measuring the low analysis among groups of people distance.

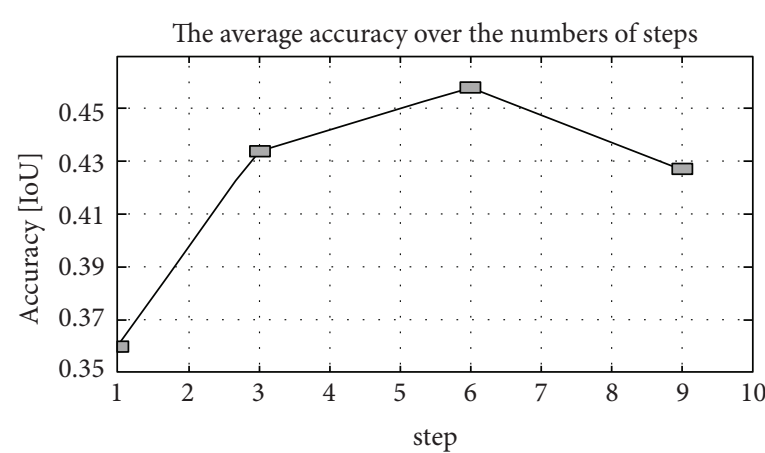

(a)

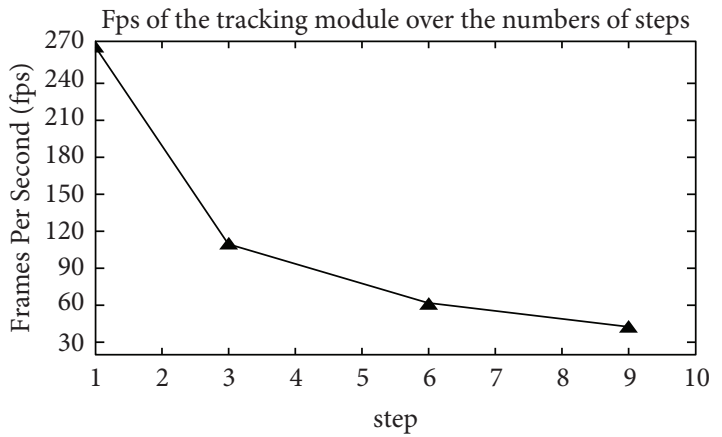

(b)

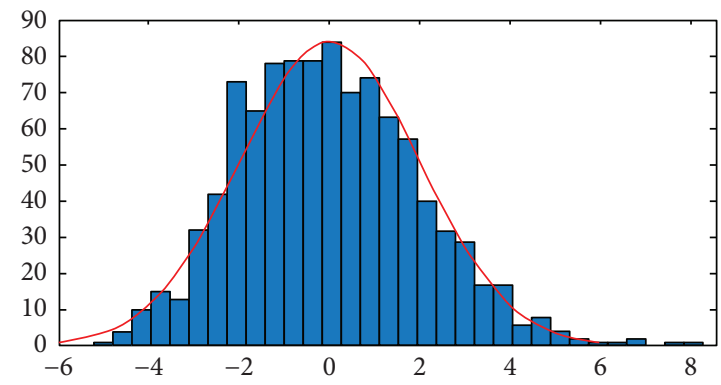

(c)

Figure 8: Accuracy chart of survivors from COVID-19 and comparative analysis on a different method. 
TABLE 2: Comparison of different social distancing models with the proposed model besides distance estimation.

\begin{tabular}{|c|c|c|c|c|c|c|c|c|}
\hline & Accuracy (\%) & Precision (\%) & Recall (\%) & Real time & Distance & Database & $\begin{array}{l}\text { Number of } \\
\text { images }\end{array}$ & $\begin{array}{c}\text { Data } \\
\text { augmentation }\end{array}$ \\
\hline Keniya et al. [39] & 92.8 & 87 & 90 & $\mathrm{Na}$ & $\mathrm{Na}$ & Auxiliary dataset & 295 & $\sqrt{ }$ \\
\hline $\begin{array}{l}\text { Sener and } \\
\text { Savarese }[40]\end{array}$ & 93.3 & 92 & 90 & $\mathrm{Na}$ & $\mathrm{Na}$ & Benchmark & $\mathrm{Na}$ & $\mathrm{X}$ \\
\hline Yadav et al. [41] & 91 & 92 & 96 & $\mathrm{Na}$ & $\mathrm{Na}$ & Face mask & 3195 & $\mathrm{X}$ \\
\hline Escalera et al. [42] & 95.6 & 96 & 96.5 & 91 & $\mathrm{Na}$ & Oxford Town & 800 & $\mathrm{X}$ \\
\hline Proposed & 97.5 & 97 & 97.5 & 95.44 & 97.88 & $\begin{array}{c}\text { UET Peshawar/Oxford } \\
\text { Town }\end{array}$ & $2200 / 800$ & $\begin{array}{c}\checkmark \\
6000 / 3200\end{array}$ \\
\hline
\end{tabular}

of UET Peshawar. Each pair was labelled, and annotation has to achieve tremendous results for the YOLOv3 proposed method. It is a real-time implementation on UET (University of Engineering and Technology), Peshawar, for proper social distancing and estimation. We augmented the database with online and offline data augmentation using different variations of image translation, image colour enhancement, image cropping, and image splitting. It increases the number of images in the Oxford Town Centre and UET Peshawar database; augmented images are 5000 and 7000, respectively. It makes the proposed method better as the previous method used a small set of databases.

A scale for defining the infection capacity and pandemic spread risk is possible through MCDM in risk analysis [43].

(i) Low infection capacity and risk measured in the range of $0 \leq \delta \leq 2.0$

(ii) Medium infection capacity and risk measured in the range of $2.0<\delta \leq 3.0$

(iii) Serious infection capacity and risk measured in the range of $3.0<\delta \leq 5.0$

(iv) High infection capacity and risk measured in the range of $5.0<\delta \leq 7.0$

(v) Very high infection capacity and risk measured in the range of $>7.0$

After defining infection capacity and pandemic spread risk, a multicriteria model was applied to select an appropriate strategic guideline for recognising health situations. Pandemics have posed severe hazards to global health and public safety in modern society. Societal order and security are important although humans are capable of defeating pandemics. Despite widespread vaccination and the use of antiviral medications, we cannot prevent the spread of the virus in the event of the next pandemic. As a result, research into pandemic prevention and control is essential. It is a popular topic all the time.

6.4. Simulation and Controllable Parameters. A simulation initiates once a stream of infested characters is hosted into a cluster of healthy individuals. The infection hurriedly spreads over the population, influences a peak when the crowd halts and rarer potential victims leave, and finally, runs its progression. The unpretentious agents in this simulation encompass a two-dimensional square world by distinct constant velocities. Some individuals are infected through a pandemic when they come nearby, passing on to others. Individuals have an age dignified in fractional time steps. Our population size was contained on $n=2000$, where $n=$ number of population batch size in the start, and we put the number of infected people $=20$, the interval between new sick individuals and every sick individual moving randomly in the area. We set birth rate $b=0.020$; it is a birth proportion directly proportional to mortality rate, and mortality $=0.50$, and in crowded places, the infection rate can increase them. Virulence $=0.020$, a distance of infectious measures each person's distance that may violate social distancing and duration depending on the infection time duration $=50$, infection time. The number of intervals individuals can measure through their speed and the violation of social distancing rate $=2$, a gap between display updates.

For social distancing and estimated results on preventive measures with the sated value of barrier to reduce the infection rate and social violation in the first phase of a barrier, we open on the barrier on the place. An intersecting barrier is divided into two zones to measure social distancing and violation results. The first half of the barrier has $70 \%$ close and $30 \%$ open in Figure 9. While in Figure 10, the results are shown with the barrier length $=0.70$. Expanding a piece of individuals in the second half, of Figure 11, we close the barrier on $100 \%$ where barrier $=100$. That achieves a massive result to control social distancing that reduces the infection rate, the death rate is shown in Figure 11 containing the barrier position, the result is given in Figure 12, and the results of social distancing and estimation tracking on real time are mentioned in Figure 6. The statistical simulation model compares the preventive measures for the setting of barrier or border position to maintain the social distancing and violation of the individual. There are six categories of characters.

Youth: in contrast, as a juvenile and adolescent, blue is innate during the simulation. Adult blue: healthy and age categorised among juveniles toward 65. Mature dark bluegreen: healthy, age over 65, clogged travelling, and staying in quarantine "at home." Sick red: the pandemic infects them, and they are inapt to enter the simulation for a specified period from the upper right at regular time intervals. Then, no more enter to infect others by passing close to them. Survivor red circles: previously infected, and after a set period, the infection in a sick individual runs its progression and becomes immune and containers no longer infect others. Expirer: act as a specified mortality rate, and sick and immune individuals are indifferent from the simulation after 

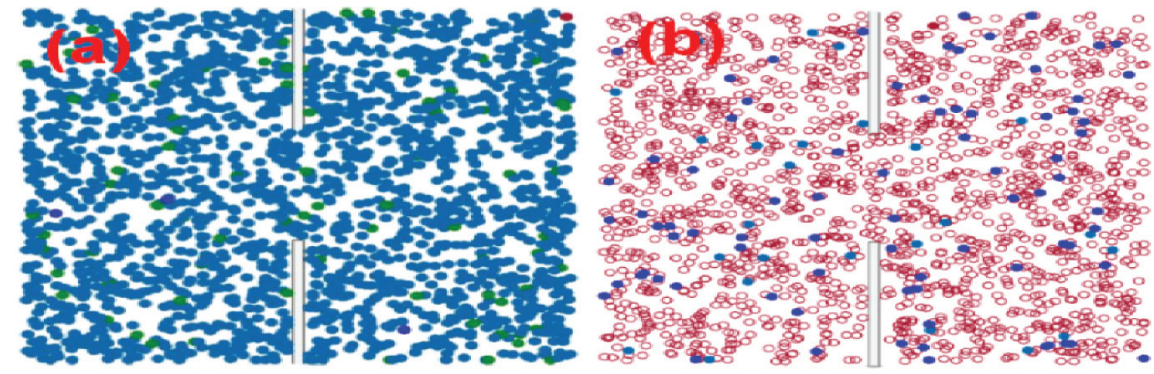

FiguRE 9: Barrier on 70\% prevention pandemic: (a) The first entered infected person from the top right red (Taftan border). (b) The overall estimation of population simulation estimated expirer rate, survivor, and transmitter moving from the first half to the second half (barrier, border, and lockdown).

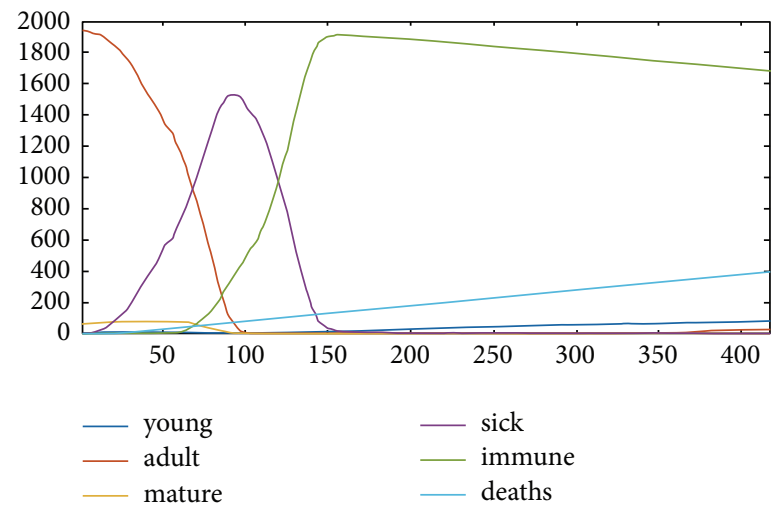

Figure 10: The number of population overall estimation of mortality and virulence prevention of the pandemic following $70 \%$ barrier.
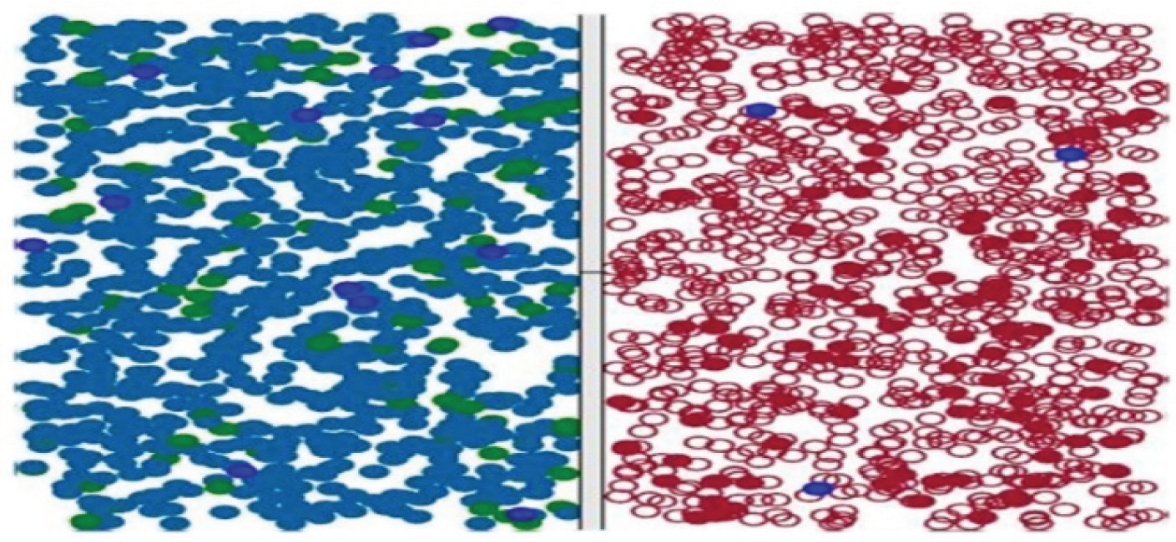

FIGURE 11: Two areas are constructed for 100 percent barrier to prevent infectious and sick from moving to another side.

end progression. Dark black: act as an expirer, and the number of removals is the death count.

The simulation originated with several sick individuals moving into a group of adults and terminates when there are no more live ill individuals. A barrier with an amendable length known as a mainstay of the simulation part splits the rectangle into two halves, as shown in Figure 9. We imagine a wall across Iran, from the Taftan border on the west side in the Baluchistan Province of Pakistan, the edge known as one of the world's most extensive borders between Pakistan and Iran. The border is the ending area of Pakistan in Baluchistan in Quetta major city known as the capital of Baluchistan. On the other hand, we imagine a wall between close cities alongside Quetta. According to the guess, 2000 people with $1 \%$ affected enter the Taftan border into Pakistan, as shown in Figure 13. 


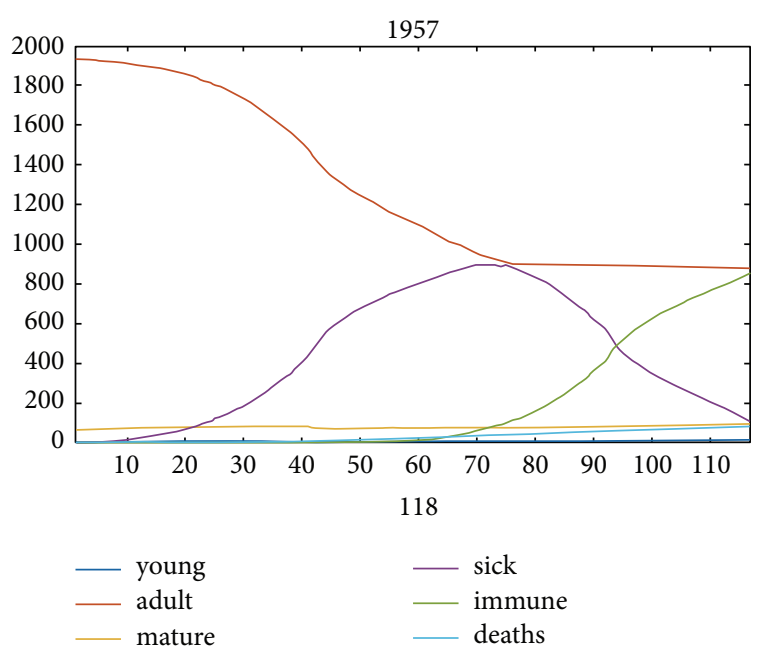

FIGURE 12: Census above the integral history of the RISTECB where the barrier is sated as a 100 percent (barrier, border, and lockdown) preventing immense dissemination and impressive control for dead and infected.

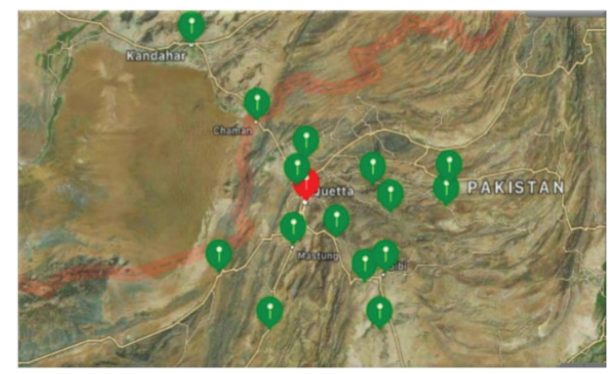

FIgUre 13: ArcGIS map of the bounding boundary between Pakistan and Iran Taftan border.

Kandahar is the nearest neighbour city in Iran that intersects with a red line border shown in Figure 13.

Young bright blue: Individuals created during the simulation

Adult blue: susceptible, on the move

Mature dark blue-green: susceptible, stay at home

Sick red: infects others when they are too close

Resister red circle: previously infected, no longer virulent

Dead infected individuals vanished from the simulation and were just counted

Expending a piece of individuals:

(i) Initiator bright blue: individuals created during the simulation

(ii) Continuer blue: suspected, on the move

(iii) Restrictor dark blue-green: susceptible, stay at home

(iv) Transmitter red: infected: infects others when they are too close

(v) Survivor red circle: previously infected, no longer virulent (vi) Expirer infected individuals are deleted from the simulation and just counted

The barrier describes the overall estimation of the population where a continuer, restrictor, and transmitter are based on infection and mortality and virulence rate in an area where the population estimated as 2000 , where several adults and young are infected; however, the infectious rate of mature is more than that of others.

The simulation in the animation runs for adjustable time steps barriers depending on the spreading epidemic and controlling the epidemic's situation. The time is on the $x$-axis, while the changing total population is in the title. It rapidly spreads through the affected area. The control barrier is stated on the 0.070, Figure 10, and the infected can quickly move from the first half to the second. Rare infected characters discover their approach through the narrow gap in the barrier. After the finished demonstration, no sick individuals were present even though no one else participated. This precise epidemic is over or setting (barrier, border, and lockdown). A diminutive ended partially of them had the infection and present immune. While 397 people are dead, virulence is the severity or harmfulness of a disease. In this simulation, virulence, a span of effectiveness, affected whether an individual passes a circle within every character. The infection distributes on the default virulence of 0.02 ; the size of the points representing the dead persons is removed from the area but counted. In Figure 10, a series of simulations investigate a wave of immense dissemination of population and numerous affected, immune, and dead.

The maximum particular run on the locale barrier on 100 percent preventing immense dissemination entire expirer from the population is 20; Figure 11 shows people's awareness about social distancing preventing immense dissemination achieved marvellous results to reduce death rate and affections. Figure 12 shows a simulation locale barrier of 100 percent decreases the essential history for death.

Attained massive results on setting (barrier, border, and lockdown) on 100 mortality rated reduced miracle prevention of real purposed effect and, hence, (Barrier, Border, and Lockdown) helps to prevent immense dissemination impressive control for expirer and transmitter. The population of 2000 inhabitants in Figure 12 depicts the number of mortality as expirer reduced in the first half of Barrier, Border, and Lockdown. It can also be seen by setting a 100 percent barrier to minimize the expirer $=20$, survivor $=792$, and sick $=22$ results of Figures 9 and 11 simultaneously. The critical point of the paper is to explain mathematically in the supple-mental electronic content. Social distancing may increase the total number in some networks of infected individuals at the end of the outbreak by simulation. Other aspects of an epidemic are the size, timing of the peak, and the length of the epidemic, which is influenced by social distancing. It appears that demonstrating some mathematical results for such more refined details of the outbreak would be difficult, but simulations can help. It is also necessary to 
perform a thorough investigation, preferably mathematical findings.

\section{Conclusions}

Social distancing measure, estimation, and COVID-19 classification with limited resources during a pandemic is the primary concern of the current situation. However, preanalysis and postanalysis of overall spreading and future aspect outcomes disaster management have a critical analysis. That is necessary to observe and stop the virus from spreading in the currently available resources. Because of the immediate attack and complicated nature of the virus, it creates difficulties to address. We proposed multidisciplinary methods to analyse future threats for social distancing measures, estimation, and feature extractions. Barriers are the critical aspect related to more attributes in the existing pandemic prevention SIER model. Therefore, the modified RISTECB model distinguishes the COVID-19 classification with barriers for social distancing measuring and estimation. It also assimilates with pre- and postaspects of simulation with locale barriers to prevent spreading.

There are also limitations to the modelling approach in the distance estimation procedure using a simple trialerror method. Everyone going outside should be in pairs to connect the framework's parameters. However, in some cases, it goes wrong with different variations of detection in the light effect, the backside of the person, and the imbalance person's postures. The identification of implementation of social distancing in these circumstances using deep learning requires proper light effects, dynamic spot, and system specification. Its limited outcomes are affecting the efficient system. Agent-based modelling techniques need more up-to-mark input to overcome predictable output with an analytical network process. However, new labelled databases and distance calibration are the limitations of the agent-based approach. The actual implementation required on different territories, light imbalance, and dynamic spots are becoming the limitations. These findings highlight the importance of region-specific considerations and ideally modelling as inputs to making policy decisions for a given region.

Simultaneous limitation of the actual tuned parameter can adhere to promising preventive and postpreventive measures. Nevertheless, the end-users are useless in monitoring the social distancing imbalance. That helps the endusers to maintain their social distance imbalance and individual characters are meaningful information for every monitoring system. Multicriteria decision-Making processes enhance the integration of the used parameters according to the situations. Single devices can help the vast organisation, territory, and healthcare centres to maintain social distancing. End-user usage of application implementation can limit the individual in proper social distancing. Every portable end-user device is helping the end-users as per different routine tasks to make sense of the limitation of the simultaneous fragment.

\section{Data Availability}

The data used to support the findings of this study are included within the article.

\section{Conflicts of Interest}

The authors declare that there are no conflicts of interest regarding the publication of this paper.

\section{Acknowledgments}

This work was supported by the National Natural Science Foundation of P. R. China (51678470 and 61803293), Natural Science Foundation of Shaanxi (2020JM-472, 2020JM-473, 2019JQ-760, 2017JM6106, and 2015JM6276), Special Research Project of the Education Department of Shaanxi Province (18JK0477), and Basic Research Foundation of Xi'an University of Architecture and Technology (JC1703 and JC1706).

\section{References}

[1] N. S. Punn, S. K. Sonbhadra, and S. Agarwal, "Monitoring COVID-19 social distancing with person detection and tracking via fine-tuned YOLO v3 and Deepsort techniques," pp. 1-10, 1927, https://arxiv.org/abs/2005.01385.

[2] Y. Zhong, "Using deep convolutional neural networks to diagnose COVID-19 from Chest X-Ray images," 2020, https:// arxiv.org/abs/2007.09695\#:\%7E:text=The\%20model\% 20validates\%20on\%20740,condition $\% 20$ of\%2095\%25\% 20confidence\%20interval.

[3] D. C. R. Novitasari, R. Hendradi, R. E. Caraka, and Y. Rachmawati, "Detection of COVID-19 chest x-ray using support vector machine and convolutional neural network," Communications in Mathematical Biology and Neuroscience, vol. 2020, 19 pages, 2020.

[4] S. K. Chandra, A. Singh, and M. K. Bajpai, "Mathematical model with social distancing parameter for early estimation of COVID-19 spread," in Proceedings of the Machine Vision and Augmented Intelligence-Theory and Applications, p. 2020, Jabalpur, India, May 2020.

[5] M. Cristani, A. D. Bue, V. Murino, F. Setti, and A. Vinciarelli, "The visual social distancing problem," IEEE Access, vol. 8, Article ID 126886, 2020.

[6] Y. Fang, H. Zhang, J. Xie et al., "Sensitivity of chest CT for COVID-19: comparison to RT-PCR," Radiology, vol. 296, no. 2, pp. E115-E117, 2020.

[7] Y. Oh, S. Park, and J. C. Ye, "Deep learning COVID-19 features on CXR using limited training data sets," IEEE Transactions on Medical Imaging, vol. 39, no. 8, pp. 2688-2700, 2020.

[8] S. Boopathi, A. B. Poma, and P. Kolandaivel, "Novel 2019 coronavirus structure, mechanism of action, antiviral drug promises and rule out against its treatment," Journal of Biomolecular Structure and Dynamics, vol. 2020, pp. 1-10, 2020.

[9] Z. Y. Zu, M. D. Jiang, P. P. Xu et al., "Coronavirus disease 2019 (COVID-19): a perspective from China,” Radiology, vol. 296, Article ID 200490, 2020.

[10] M. Loey, F. Smarandache, and N. E. M. Khalifa, "Within the lack of chest COVID-19 X-ray dataset: a novel detection model based on GAN and deep transfer learning," Symmetry, vol. 12, no. 4, p. 651, 2020. 
[11] M. M. Saito, S. Imoto, R. Yamaguchi et al., "Extension and verification of the SEIR model on the 2009 influenza A (H1N1) pandemic in Japan," Mathematical Biosciences, vol. 246, no. 1, pp. 47-54, 2013.

[12] R. Peng and F. Yi, "Asymptotic profile of the positive steady state for an SIS epidemic reaction-diffusion model: effects of epidemic risk and population movement," Physica D: Nonlinear Phenomena, vol. 259, pp. 8-25, 2013.

[13] D. K. Das, R. Govindan, I. Nikić-Spiegel, F. Krammer, E. A. Lemke, and J. B. Munro, "Direct visualization of the conformational dynamics of single influenza hemagglutinin trimers," Cell, vol. 174, no. 4, pp. 926-937, 2018.

[14] J. Li, Y. Wang, J. Wu et al., "Do stay at home orders and cloth face coverings control COVID-19 in New York city? Results from a SIER model based on real-world data," Open Forum Infectious Diseases, vol. 8, no. 2, 2020.

[15] Y.-C. Chen, P.-E. Lu, C.-S. Chang, and T.-H. Liu, "A timedependent SIR model for COVID-19 with undetectable infected persons," IEEE Transactions on Network Science and Engineering, vol. 7, no. 4, pp. 3279-3294, 2020.

[16] F. Ganem, "Monitoring social distancing and SARS-CoV-2 transmission in brazil using cell phone mobility data affiliations," Preprint, SSRN Electronic Journal, 2020.

[17] G. Eng, "Initial model for the impact of social distancing on CoVID-19 spread," Preprint, 2020.

[18] C. Butt, J. Gill, D. Chun, and B. A. Babu, "Deep learning system to screen coronavirus disease 2019 Pneumonia," 2020, https://arxiv.org/abs/2002.09334.

[19] I. Cooper, A. Mondal, C. G. Antonopoulos, A. Mondal, and C. G. Antonopoulos, "A SIR model assumption for the spread of COVID-19 in different communities," Chaos, Solitons \& Fractals, vol. 139, Article ID 110057, 2020.

[20] K. Prem, Y. Liu, T. W. Russell, A. J. Kucharski, R. M. Eggo, and N. Davies, "Articles the effect of control strategies to reduce social mixing on outcomes of the COVID-19 epidemic in Wuhan, China: a modelling study," vol. 5, no. 5, pp. 261-270, 2020.

[21] L. Sarker, M. M. Islam, T. Hannan, Z. Ahmed, Z. Ahmed, and A. Zakaria, "Covid-DenseNet: COVID-DenseNet: a deep learning architecture to detect COVID-19 from chest radiology images," Preprints, no. 5, 2020.

[22] C. Adolph, K. Amano, B. Bang-Jensen, N. Fullman, and J Wilkerson, "Pandemic politics: timing state-level social distancing responses to COVID-19," Journal of Health Politics Policy and Law, vol. 46, pp. 1-19, 2020.

[23] C. E. Walters, K. E. C. Ainslie, H. Fu et al., "Evidence of initial success for China exiting COVID-19 social distancing policy after achieving containment," no. 5, pp. 1-10, 2020.

[24] A. B. Gumel, E. A. Iboi, C. N. Ngonghala, and E. H. Elbasha, "A primer on using mathematics to understand COVID-19 dynamics: modeling, analysis and simulations," Infectious Disease Modelling, vol. 6, pp. 148-168, 2021.

[25] E. F. Doungmo Goufo, R. Maritz, and J. Munganga, "Some properties of the Kermack-McKendrick epidemic model with fractional derivative and nonlinear incidence," Advances in Difference Equations, vol. 2014, no. 1, 9 pages, 2014.

[26] P. Majumder, P. Biswas, and S. Majumder, "Application of new topsis approach to identify the most significant risk factor and continuous monitoring of death of COVID-19," Electronic Journal of General Medicine, vol. 17, no. 6, 2020.

[27] A. Mohammed, M. Yazdani, A. Oukil, and E. D. R. Santibanez Gonzalez, "A hybrid MCDM approach towards resilient sourcing," Sustainability, vol. 13, no. 5, p. 2695, 2021.
[28] Z. Yang, X. Li, H. Garg, and M. Qi, "Decision support algorithm for selecting an antivirus mask over COVID-19 pandemic under spherical normal fuzzy environment," International Journal of Environmental Research and Public Health, vol. 17, no. 10, p. 3407, 2020.

[29] N. Alkan and C. Kahraman, "Evaluation of government strategies against COVID-19 pandemic using q-rung orthopair fuzzy TOPSIS method," Applied Soft Computing, vol. 110, Article ID 107653, 2021.

[30] A. C. Boyacı and A. Şişman, "Pandemic hospital site selection: a GIS-based MCDM approach employing Pythagorean fuzzy sets," Environmental Science and Pollution Research, vol. 19, 2021.

[31] T. Ozturk, M. Talo, E. A. Yildirim, U. B. Baloglu, O. Yildirim, and U. Rajendra Acharya, "Automated detection of COVID19 cases using deep neural networks with X-ray images," Computers in Biology and Medicine, vol. 121, Article ID 103792, 2020.

[32] K. El Asnaoui, Y. Chawki, and A. Idri, "Automated methods for detection and classification pneumonia based on $\mathrm{x}$-ray images using deep learning,"vol. 90, Springer, 2020, https:// arxiv.org/abs/2003.14363.

[33] J. Liu, K. M. Kamarudin, Y. Liu, and J. Zou, "Developing pandemic prevention and control by anp-qfd approach: a case study on urban furniture design in China communities," International Journal of Environmental Research and Public Health, vol. 18, no. 5, pp. 2653-2726, 2021.

[34] H. Sarmadi and A. Karamodin, "A novel anomaly detection method based on adaptive Mahalanobis-squared distance and one-class kNN rule for structural health monitoring under environmental effects," Mechanical Systems and Signal Processing, vol. 140, Article ID 106495, 2020.

[35] A. P. Lemos-pai, Since January 2020 Elsevier Has Created a COVID-19 Resource centre with Free Information in English and Mandarin on the Novel Coronavirus COVID- 19. The COVID-19 Resource centre Is Hosted on Elsevier Connect, the company's public news and information, Elsevier, Amsterdam, Netherlands, 2020.

[36] J. Su, X. He, L. Qing, T. Niu, Y. Cheng, and Y. Peng, “A novel social distancing analysis in urban public space: a new online spatio-temporal trajectory approach," Sustainable Cities and Society, vol. 68, Article ID 102765, 2021.

[37] S. Bharati, P. Podder, and M. R. H. Mondal, "Hybrid deep learning for detecting lung diseases from X-ray images," Informatics in Medicine Unlocked, vol. 20, Article ID 100391, 2020.

[38] A. Ullah, H. Elahi, Z. Sun, A. Khatoon, and I. Ahmad, "Comparative analysis of AlexNet, ResNet18 and SqueezeNet with diverse modification and arduous implementation," Arabian Journal for Science and Engineering, vol. 65, 2021.

[39] R. Keniya, A. Khakharia, V. Shah et al., "Disease prediction from various symptoms using machine learning," SSRN Electronic Journal, 2020.

[40] O. Sener and S. Savarese, "Active learning for convolutional neural networks: a core-set approach," in Proceedings of the 6th International Conference on Learning Representations, pp. 1-13, Vancouver, Canada, May 2018.

[41] U. N. Yadav, O. P. Yadav, D. R. Singh et al., "Perceived fear of COVID-19 and its associated factors among Nepalese older adults in eastern Nepal: a cross-sectional study," PLoS One, vol. 16, no. 7, pp. e0254825-15, 2021.

[42] S. Escalera, M. A. Bagheri, M. Valstar et al., "ChaLearn looking at people and faces of the world: face AnalysisWorkshop and challenge 2016," in Proceedings of the 2016 IEEE Conference on Computer Vision and Pattern Recognition 
Workshops (CVPRW), pp. 706-713, IEEE, Las Vegas, NV, USA, June2016.

[43] D. Pamučar, M. Žižović, D. Marinković, D. Doljanica, S. V. Jovanović, and P. Brzaković, "Development of a multicriteria model for sustainable reorganization of a healthcare system in an emergency situation caused by the COVID-19 pandemic," Sustainability, vol. 12, no. 18, pp. 7504-7524, 2020. 